\title{
Methodologische Entwicklungen in der Gewaltforschung.
}

\author{
Videodatenanalyse, Mixed Methods und Big Data
}

\author{
Anne Nassauer $\cdot$ Nicolas M. Legewie
}

(C) Der/die Autor(en) 2020

Zusammenfassung Über Jahrzehnte wurde Gewalt durch Interviews mit Betroffenen oder Tätern, durch teilnehmende Beobachtung oder Gewaltstatistiken untersucht, meist unter Verwendung entweder qualitativer oder quantitativer Analysemethoden. Seit der Jahrhundertwende stehen Forschenden eine Reihe neuer Ansätze zur Verfügung: Es gibt immer mehr Videoaufnahmen von gewaltsamen Ereignissen, Mixed Methods-Ansätze werden stetig weiterentwickelt und durch Computational Social Sciences finden Big Data-Ansätze Einzug in immer mehr Forschungsfelder. Diese drei Entwicklungen bieten großes Potenzial für die quantitative und qualitative Gewaltforschung. Der vorliegende Beitrag diskutiert Videodatenanalyse, Triangulation und Mixed Methods-Ansätze sowie Big Data und bespricht den gegenwärtigen und zukünftigen Einfluss der genannten Entwicklungen auf das Forschungsfeld. Das Augenmerk liegt besonders darauf, (1) wie neuere Videodaten genutzt werden können, um Gewalt zu untersuchen und wo ihre Vor- und Nachteile liegen, (2) wie Triangulation und Mixed Methods-Ansätze umfassendere Analysen und theoretische Verknüpfungen in der Gewaltforschung ermöglichen und (3) wo Anwendungen von Big Data und Computational Social Science in der Gewaltforschung liegen können.

Schlüsselwörter Gewaltforschung · Videodaten · Mixed Methods · Big Data • Computational Social Sciences

\footnotetext{
A. Nassauer $(\bowtie)$

John-F.-Kennedy-Institut, Abteilung Soziologie, Freie Universität Berlin, Lansstraße 7-9, 14195 Berlin, Deutschland

E-Mail: anne.nassauer@fu-berlin.de

N. M. Legewie $(\bowtie)$

Department of Sociology, University of Pennsylvania, Locust Walk, PA 3718, USA

E-Mail: NLegewie@diw.de
} 


\title{
Methodological developments in violence research
}

Video data analysis, mixed methods, and big data

\begin{abstract}
For decades violence research has relied on interviews with victims and perpetrators, on participant observation, and on survey methods, and most studies focused on either qualitative or quantitative analytic strategies. Since the turn of the millennium, researchers can draw on a range of new approaches: there are increasing amounts of video data of violent incidents, triangulation and mixed methods approaches become ever more sophisticated, and computational social sciences introduce big data analysis to more and more research fields. These three developments hold great potential for quantitative and qualitative violence research. This paper discusses video data analysis, mixed methods, and big data in the context of current and future violence research. Specific focus lies on (1) potentials and challenges of new video data for studying violence; (2) the role of triangulation and mixed methods in enabling more comprehensive violence research from multiple theoretical perspectives, and (3) what potential uses of big data and computational social science in violence research may look like.
\end{abstract}

Keywords Violence research · Video data $\cdot$ Triangulation $\cdot$ Mixed methods $\cdot$ Big data $\cdot$ Computational social science

\section{Einleitung}

Technische Fortschritte seit der Jahrtausendwende veränderten die Forschungswelt grundlegend. Mixed Methods und Big Data wurden zu Schlagwörtern und Computational Social Sciences sowie die Zunahme von Videodaten ergänzen und verändern etablierte Forschungsansätze. Diese Entwicklungen machen auch vor der Gewaltforschung nicht halt, wurden jedoch bisher kaum zusammenfassend diskutiert. Der vorliegende Beitrag untersucht daher den Einfluss und die Potenziale neuerer technologischer und methodologischer Entwicklungen auf das Forschungsfeld. Er diskutiert (1) wie neuere Videodaten genutzt werden können, um Gewalt zu untersuchen und wo die Vor- und Nachteile für die Gewaltforschung liegen, (2) wie Triangulation und Mixed Methods-Ansätze in der Videodatenanalyse wichtige Beiträge zur Gewaltforschung liefern können und (3) wo Anwendungen im Bereich von Big Data und Computational Social Sciences liegen können. Er argumentiert, dass (1) neuere Videodaten vielversprechende Einblicke in Gewalthandlungen und neue Perspektiven auf Gewaltentstehung ermöglichen, jedoch nicht für jede Fragestellung sinnvoll sind, dass (2) Triangulation und Mixed Methods-Studien enorme Möglichkeiten für umfassende Gewaltanalysen sowie für die Verknüpfbarkeit von situativen und Kontext-Erklärungen bieten und dass (3) Big Data und Computational Social Sciences bestehende Ansätze zur Erforschung von Gewalt unterstützen und neue Ansätze hervorbringen können. Zusammen eröffnen diese neuen methodischen Ansätze der Gewaltforschung enorme Möglichkeiten und versprechen so einen erheblichen Innovationsschub im Forschungsfeld. 


\section{Videodatenanalyse in sozialwissenschaftlicher Gewaltforschung}

\subsection{Neuere Videodaten eröffnen ungeahnte Möglichkeiten}

Immer mehr Menschen nutzen neue Technologien, um Handlungen, Interaktionen und Ereignisse im Alltag zu filmen. Mobiltelefone ermöglichen es, technisch immer hochwertigere Videos zu drehen und werden von vielen Menschen fast permanent mit sich geführt. Überwachungskameras und Body-Cameras werden weitläufig eingesetzt und mit Kameras ausgestattete Drohnen finden sich in immer mehr Haushalten. Eine Konsequenz dieser Entwicklung ist, dass Menschen sich häufiger und in immer mehr Situationen filmen und filmen lassen. Heute werden nicht nur Hochzeiten aufgezeichnet, sondern auch Beerdigungen und Geburten, nicht nur alltägliches Verhalten im öffentlichen Raum, sondern auch Unfälle, Massenpaniken und gewaltsame Übergriffe. Nicht mehr nur Familienfeiern und Reisen in ferne Länder werden für den direkten Freundeskreis gefilmt, sondern auch Vergewaltigungen, Morde und Suizide wurden bereits aufgezeichnet, um sie mit Millionen potenzieller Zuschauer*innen im Internet zu teilen. Extreme Auswüchse dieser Entwicklung sind die live übertragenen terroristischen Anschläge auf zwei Moscheen in Christchurch, Neuseeland, im März 2019 und auf eine Synagoge in Halle, Deutschland, im Oktober 2019.

In der Vergangenheit musste sich die Gewaltforschung häufig auf die Schilderungen von Betroffenen und Täter*innen verlassen, um Informationen über Gewaltsituationen zu sammeln und zu untersuchen, warum Gewalt ausbrach. Opfer von Gewalthandlungen sind jedoch oft traumatisiert und können sich meist nur bruchstückhaft und selektiv erinnern. Täter*innen neigen in Interviews dazu anzugeben oder zu übertreiben und haben zudem ebenfalls Schwierigkeiten, sich an Details zu erinnern. In beiden Fällen können soziale Erwünschtheit und rückblickende Rechtfertigungen Daten verzerren und den Details, die Menschen wahrnehmen und an die sie sich erinnern können, sind klare Grenzen gesetzt (Bernard et al. 1984; Nassauer und Legewie 2018: $6 \mathrm{ff}$.; Vrij et al. 2014). ${ }^{1}$ All dies schränkt die Brauchbarkeit von qualitativen und Survey-Interviews als primäre Datenquellen für die Untersuchung von Gewaltsituationen erheblich ein. Es ist aber auch keine Alternative für Forschende, an einem öffentlichen Platz teilnehmende Beobachtungen durchzuführen in der „Hoffnung“, eine Gewalthandlung möge ausbrechen - abgesehen von forschungsethischen Fragen ist ein solches Vorgehen in der Durchführung schon deshalb schwierig, weil Gewaltereignisse zu selten sind, um sie auf diese Weise systematisch beobachten zu können. Und selbst wenn dem nicht so wäre, so stünden Forschende doch vor dem gleichen Problem wie Betroffene in gewaltsamen Situa-

\footnotetext{
1 Ein Extrembeispiel ist der terroristische Anschlag in einem Einkaufszentrum in Nairobi im Jahr 2013, bei dem die Täter*innen 67 Menschen töteten und sich mehrere Tage im Einkaufszentrum verbarrikadierten, während sich dort noch Menschen versteckten und zu fliehen versuchten. Menschen, die entkommen konnten, hatten einen starken Anreiz, der Polizei und den Medien möglichst akkurate Informationen über Tathergang und Täter*innen zu liefern. Oft waren Angehörige oder Freunde der Fliehenden selbst noch im belagerten Einkaufszentrum. Dennoch zeigte eine spätere Auswertung der Überwachungskamera-Videos, dass die meisten Details, die fliehende Opfer an Polizei weitergaben, nicht mit bestimmten Tatsachen, wie der Anzahl der Täter*innen, übereinstimmten (Gatehouse 2013).
} 
tionen: Sie könnten Situationen nicht in Gänze wahrnehmen, sich üblicherweise aufgrund von emotionaler Erregung und Stressreaktionen nicht im Detail erinnern und als Daten lediglich ihre Feldnotizen mit der Wissenschaftsgemeinschaft und der Öffentlichkeit teilen (Nassauer und Legewie 2018: 6 ff.).

Videodaten ermöglichen hier andere Ansätze. Selbst wenn nur ein Bruchteil der exponentiell zunehmenden Aufzeichnungen von Smartphones, Überwachungskameras (CCTV) und Body-Cameras Gewalthandlungen zeigt, kann die Gewaltforschung immer noch auf Millionen von Videos für ihre Analysen zurückgreifen. Solche Videoaufzeichnungen von Gewalthandlungen ermöglichen es Forschenden Gewaltsituationen „vom Schreibtisch aus“ zu beobachten, Details in Zeitlupe zu erfassen und durch Abspielen in doppelter Geschwindigkeit Interaktions-Rhythmen von Situationen zu analysieren. Sie ermöglichen auch, Primärdaten zu teilen und z. B. eine Situation von mehreren Forschenden analysieren zu lassen, um Reliabilitätstests durchzuführen (Campbell et al. 2013). So kann bspw. die Kodierung und Analyse von Videodaten durch mehrere Forschende überprüft werden, um so die Verlässlichkeit der Kodierung in qualitativen und quantitativen Studien zu erhöhen (für ein Beispiel aus der quantitativen Videoanalyse siehe u. a. Levine et al. 2011).

Diese Entwicklungen erlauben erstmals die empirische Überprüfung einiger zentraler theoretischer Weiterentwicklungen in der Gewaltforschung. Z. B. werden Kontextfaktoren regelmäßig ex post facto als Rechtfertigungsgrund für gewaltsames Handeln herangezogen und in Zeitungsartikeln hervorgehoben oder in Interviews von Täter*innen oder Betroffenen genannt. Seit den 1980er Jahren nimmt die soziologische Forschung aber zunehmend an, dass Missstände und andere Kontextfaktoren nicht erklären können, ob, wann und wo Gewalt entsteht. Gleiches gilt für Motivationen von Akteuren*innen: Die meisten Personen wenden trotz Kontextfaktoren und etwaiger Motivation oder Aggression keine Gewalt an (siehe u. a. Athens 1980; Felson und Steadman 1983; Stott und Reicher 1998; Wright und Decker 1997). Lange waren Forschende mangels passender Daten jedoch nicht in der Lage, situative Dynamiken systematisch zu untersuchen und ihren Einfluss mit dem von Kontextfaktoren und Motivationen zu vergleichen. Diese Beschränkung wurde durch Videodaten überwunden, die es heute ermöglichen, nicht nur Kontextfaktoren für Gewalt, sondern auch situative Faktoren vergleichend zu analysieren.

Videodaten werden seither zum einen als kulturelles Artefakt und Gegenstand von Interaktionen und sozialen Prozessen untersucht. So zeigt bspw. Goodwin (1994), wie Videos in Gerichtsverhandlungen verwendet werden, um Narrative zu konstruieren und beschreibt die Wahrnehmung und Interpretation von Videos als sozialen Prozess. Watson (2018) arbeitet mit einem ähnlichen Ansatz und untersucht, wie eine Videoaufzeichnung in einem Mordprozess in Kanada genutzt wurde, um die Erschießung eines Zivilisten durch einen Polizeibeamten von Mord mit bedingtem Vorsatz in versuchten Mord umzudeuten. Tuma (2017) untersucht, wie Videos in polizeilichen Untersuchungen gehandhabt werden und beschreibt speziell das Beispiel einer Untersuchung zu gewaltsamen Auseinandersetzungen während eines Großereignisses. Ferrándiz und Baer (2008) beschreiben in ihrer Studie, wie visuelle Medien, die von Teilnehmenden an Ausgrabungen der Opfer des Franko-Regimes in Spanien aufgenommen werden, als „digitales Gedächtnis und Erinnern“ fungieren. 
Seit etwa der Jahrtausendwende werden Videodaten aber auch als Datenträger für die Untersuchung von Gewaltsituationen selbst verwendet. In diesem Artikel fokussieren wir uns auf diesen letzteren Anwendungsbereich. Die Verwendung von Videodaten als Blickfenster in Gewaltsituationen hinein geschieht aktuell über viele Disziplinen hinweg mit dem Ziel, Gewalthandlungen auf ihre Entstehung und Ursachen hin zu untersuchen. Sie finden zunehmende Verwendung in der Soziologie (Collins 2008; Klusemann 2009, 2012; Nassauer 2019b), Psychologie (Levine et al. 2011; Philpot et al. 2019; Philpot und Levine 2016), Politikwissenschaft (Bramsen 2018) und Kriminologie (Lindegaard et al. 2015; Mastrofski et al. 2010; Mosselman et al. 2018; Nassauer 2018a). ${ }^{2}$ Mithilfe von Videodaten liefern diese Studien neue Erkenntnisse und tragen zum Verständnis sozialer und kultureller Prozesse und Ereignisse bei. So zeigt Klusemann (2009) bspw. erstmals die Bedeutung situativer emotionaler Dynamiken für die Entstehung von Massakern. Levine et al. (2011; siehe auch Philpot et al. 2019) beschreiben den Einfluss situativer Interaktionen auf den Ausbruch oder die Schlichtung von Schlägereien und Nassauer (2019b) untersucht, wie situative Interaktionen, Interpretationen und emotionale Dynamiken zum Ausbruch von Protestgewalt führen können. Collins (2008) zeigt, wie wesentlich situative Dynamiken u. a. für terroristische Anschläge sind und Lindegaard, Bernasco und Jacques (2015) zeichnen die Rolle situativer Dynamiken für Gewalthandlungen in Raubüberfällen nach (siehe auch Liebst et al. 2019; Mosselman et al. 2018).

Die zunehmende Verwendung von Videos aus dem realen Leben als Datenquelle zur Erforschung situativer Dynamiken von Gewalt (und anderen Phänomenen) erfordert eine Weiterentwicklung bestehender Methoden der Video- und Situationsanalyse. Gewaltforschende verfügten über keinen klaren methodologischen Bezugsrahmen, kaum Werkzeuge und Gütekriterien zur systematischen Analyse dieser neuen Videodaten. Bestehende Ansätze liefern einige wichtige Ausgangspunkte: teilnehmende Beobachtung (z.B. Atkinson et al. 2007), multimodale Interaktionsanalyse (z.B. Norris 2004), experimentelle Videodaten aus psychologischen Experimenten (z.B. Gross und Levenson 1997; Elsner und Wertz 2019) oder interpretative Videoanalyse (z.B. Heath et al. 2010; Knoblauch et al. 2008; Pauwels 2015; Moritz und Corsten 2018). Allerdings bietet keiner der Ansätze einen vollständigen Werkzeugkasten für die Analyse von Handlungen und Situationsdynamiken, die zu spezifischen sozialen Ereignissen oder Prozessen führen (Nassauer und Legewie 2018: 4 ff.). Nassauer und Legewie $(2018,2019)$ schlagen ein solches methodologisches Gerüst zur Verwendung von Videodaten vor, aufbauend auf etablierten Ansätzen und dem Vorgehen von Forschenden in bestehenden Studien: Video Data Analysis (VDA). VDA zielt speziell darauf ab, einen interdisziplinären methodologischen Rahmen zu liefern, hilfreiche Perspektiven und Werkzeuge zur Analyse neuerer Videodaten zur Verfügung zu stellen und den interdisziplinären Austausch zur situativen Videoanalyse zu fördern. VDA formuliert dazu einen Werkzeugkasten analytischer Dimensionen und Analyseprozesse sowie Kriterien für Reliabilität ( $\mathrm{Zu}-$ verlässigkeit der Messung), Validität (Gültigkeit der Messung), Transparenz (Nachvollziehbarkeit der Forschung) und Forschungsethik. Je nach Forschungsinteresse

\footnotetext{
${ }^{2}$ Für eine historische Perspektive der Nutzung von Videodaten in den Sozialwissenschaften siehe Collins (2019a), Dingwall (2019), oder Erickson (2011).
} 
und theoretischem Ansatz können bestimmte Aspekte einer oder mehrerer analytischer Dimensionen im Fokus stehen: Gesicht und Körpersprache, Interaktionen und Handlungssequenzen sowie physischer und sozialer Kontext der Situation. Diese Dimensionen sollten als analytische Brillen verstanden werden, die helfen können, Videodaten zu analysieren. Analyseschritte umfassen das Kodieren des Videomaterials, die Rekonstruktion von Timing und zeitlichen Abläufen und, soweit möglich und gewollt, die Erfassung situativer Zusammenhänge.

Aufgrund des relativ großen Aufwands der detaillierten Analyse von Videodaten nutzen die meisten VDA-Studien bislang kleine und mittlere Fallzahlen. Es ist daher wichtig zu betonen, dass VDA einen ,agnostischen“ Analyserahmen bietet, der sowohl für qualitative als auch für quantitative Analysen genutzt werden kann (z.B. Levine et al. 2011; Nassauer 2018a). Dementsprechend kann das Kodieren sowohl induktiv oder deduktiv geschehen und mehr oder weniger interpretativ erfolgen (für Details siehe Nassauer und Legewie 2019). Dieser variable Einsatz kann am Beispiel tödlicher Polizeigewalt gegen afro-amerikanische Bürger*innen veranschaulicht werden: VDA kann für detaillierte Fallanalysen verwendet werden, in denen potenziell rassistische Interpretationen, Interaktionsdynamiken und Deutungsmuster rekonstruiert werden, um die Wirkungsweisen zu verstehen, durch die Hintergrundfaktoren (wie symbolischer Rassismus) und situative Faktoren (Interaktion, Interpretation und Emotion) zu Erschießungen führen (Nassauer 2020). VDA kann aber auch auf große Datensätze angewandt werden: In solchen würden einzelne Einsätze per VDA kodiert und der Datensatz mit statistischen Verfahren ausgewertet. Z.B. arbeiten McCluskey, Uchida und Kolleg*innen mit einem Datensatz von potenziell knapp 10 Mio. Body-Camera-Videos des Los Angeles Police Department, LAPD (McCluskey et al. 2019). In einem solchen Datensatz könnte z. B. quantitativ untersucht werden, ob Zusammenhänge zwischen spezifischen situativen Faktoren (das Opfer widersetzt sich der Festnahme, das Opfer kehrt das Gesicht von den Polizist*innen ab, das Opfer zieht eine Waffe) und Kontextfaktoren (Einsatzraum, Gewaltrate im Polizeiabschnitt, Erschießung von Polizist*innen in jüngerer Vergangenheit) bestehen. Eine quantitative Analyse von situativen Faktoren zur Gewalt wurde bspw. von Levine et al. (2011) durchgeführt und wird, speziell für große Datensätze, wie den der LAPD, mit Fortschritten in der automatisierten Videoanalyse zunehmend an Bedeutung gewinnen (siehe unten).

\subsection{Gütekriterien und forschungsethische Überlegungen}

Je nach verwendetem Forschungsansatz können in der videobasierten Gewaltforschung etablierte Standards für Gütekriterien und Forschungsethik verwendet werden (z. B. Knoblauch et al. 2008; King et al. 1994; Wiles et al. 2011). Z. B. könnten quantitative Analysen des Datensatzes von Uchida und Kolleg*innen auf ihre Repräsentativität für Interaktionen des LAPD mit Bürger*innen hin geprüft werden, während qualitative Analysen mit dem Konzept der theoretischen Sättigung die Fallauswahl bewerten könnten. Teilweise erfordert die Videodatenanalyse neue 
oder angepasste Perspektiven auf Validität und forschungsethische Gesichtspunkte (Nassauer und Legewie 2018; Legewie/Nassauer und Legewie 2018, 2019). ${ }^{3}$

Als Kriterien zur Validität schlagen wir (2019) drei Kernaspekte vor: Erstens sollten Forschende zur Rekonstruktion und Analyse situativer Dynamiken, Ereignisse und Prozesse auf die Verwendung neutraler oder ausgewogener Quellen achten. Die Quellen, von denen Videodaten bezogen werden, sollten der analysierten Situation möglichst neutral und ohne spezielle Interessenlage gegenüberstehen. Falls dem nicht so ist, sollten verschiedene Datenquellen trianguliert werden (bspw. Daten der Polizei und von Demonstrierenden; siehe unten). Ein zweites Gütekriterium ist die optimale Erfassung des analysierten Ereignisses oder Prozesses. Optimale Erfassung bedeutet, dass die visuellen Daten (bzw. eine Kombination aus visuellen und anderen Datentypen, siehe unten) die Situation oder das Ereignis und alle involvierten Akteur*innen möglichst komplett einfangen sollte. Da häufig von Interesse ist, wie das Ereignis im Detail abläuft, sollten keine Informationen fehlen, die für diesen Ablauf maßgeblich sind (z.B., weil Akteur*innen das Bild verlassen oder Videoaufzeichnungen geschnitten werden und so Handlungs- oder Prozessabläufe in der Aufzeichnung fehlen). Drittens sollte Videomaterial auf natürliches Verhalten überprüft werden (Harper 1988, S. 28; Pauwels 2010, S. 553 f.; Wagner 2002, S. 165). Natürliches Verhalten bedeutet, dass die gefilmte Person sich ohne die Videoaufnahme nicht anders verhalten hätte. Drei Fragen können Forschenden dabei helfen, sich ein Bild zu machen (Nassauer und Legewie 2018, S. 23): (a) Waren sich gefilmte Personen bewusst, dass sie gefilmt wurden? (b) Wenn ja, gibt es Hinweise, dass sie ihr Verhalten angepasst haben? (c) Wenn ja, inwiefern ist Reaktivität auf Videokameras Teil der Situation und kann als natürliches Verhalten gesehen werden (d.h., das Interaktionsritual schließt Kameras mit ein, wie bspw. in den meisten Demonstrationen, die für die Öffentlichkeit und Medien stattfinden und in denen Filmaufnahmen daher üblich sind)?

Neben der Überprüfung solcher Gütekriterien erfordern neuere Arten von Videodaten auch einen neuen Blick auf Fragen der Forschungsethik. Forschungsethik bezeichnet die Anwendung ethischer Prinzipien im Forschungsprozess. Zu den Grundsätzen der Forschungsethik gehört es, Studien-Teilnehmende vor Schaden zu bewahren, Interessenskonflikte und Falschdarstellungen zu vermeiden, geltendes Recht zu respektieren und professionelle Standards, wie wissenschaftliche Kompetenz und Nicht-Diskriminierung, zu befolgen. Diese Grundprinzipien werden in einer Vielzahl allgemeiner Richtlinien für ethische Sozialforschung ausdifferenziert (z. B. ASA 2018; BSA 2017b; DGS/BDS 2017; RatSWD 2017). Überwachungskamera-Videos von Schlägereien, auf YouTube hochgeladene Mobiltelefonaufnahmen kollektiver Gewalt, Body-Camera-Videos von Polizeigewalt oder Livestreams von Amokläufen sind jedoch eine relativ neue Datenform. Und während in der Forschung neben Richtlinien zu allgemeiner Forschungsethik bereits Auseinandersetzungen mit ethischen Gesichtspunkten visueller Daten (Pauwels 2006; Wiles et al. 2008, 2011) und Online-Daten (BSA 2017a; Markham und Buchanan 2012; Zimmer 2010) zu finden sind, wurden diese neueren Video-Datentypen und die ethischen Implikationen

\footnotetext{
${ }^{3}$ Hinsichtlich Fragen der Reliabilität unterscheiden sich Videodaten hingegen nicht grundlegend von anderen Datentypen (Legewie et al. 2019, S. 15).
} 
ihrer Nutzung in der Forschung bisher weniger besprochen. Basierend auf bestehenden Diskussionen zu sozialwissenschaftlicher Ethik sollten daher Studien, die diese neueren Arten der Videodaten nutzen, vor dem Hintergrund von fünf Kernaspekten der Forschungsethik bewertet werden (Legewie und Nassauer 2018, 2020; Pauwels 2006; Wiles et al. 2011): (1) informierte Einwilligung, (2) Schutz der Privatsphäre, (3) einzigartige Forschungsmöglichkeiten durch Datennutzung, (4) Minimierung möglichen Schadens für Teilnehmende und (5) Transparenz der Forschung. Diese ethischen Prinzipien sollten von Studie zu Studie im jeweiligen Forschungskontext gegeneinander aufgewogen und bewertet werden. Eine Studie mag bspw. in den ersten zwei Aspekten als ethisch problematisch, in den letzten dreien aber positiv bewertet werden. Forschende können anhand dieser Bewertung abwägen, ob eine Studie durchgeführt werden soll und ob Anpassungen angebracht sind, um forschungsethisch problematische Punkte zu beheben oder abzuschwächen (Salganik 2018, S. 196ff.). Je nach Studie können Forschende bei der Gesamtbewertung auch einzelnen der fünf Aspekte mehr oder weniger Gewicht zusprechen. Wesentlich ist eine transparente und begründete Auswertung der ethischen Gesichtspunkte vorzunehmen.

\subsection{Die Grenzen video-basierter Gewaltforschung}

Wir stehen also vor immer mehr Daten zur Erforschung von Gewalt, die uns eine Vielzahl neuer Einblicke in Gewalthandlungen bieten können, sofern wir Gütekriterien und ethische Gesichtspunkte berücksichtigen. Solche neueren Arten von Videodaten und Videodatenanalysen sind allerdings nicht für jede Fragestellung der Gewaltforschung geeignet. Der Ansatz (vor allem in Kombination mit Dokumentdaten, siehe unten) bietet sich bspw. an, wenn Forschende untersuchen wollen, wie und warum verschiedene Formen der Gewalt - von individuellen Übergriffen zu kollektiver Gewalt - entstehen, wie Personen in Gewalthandlungen eingreifen und wie effektiv verschiedene Arten der De-Eskalation sind. Sofern eine Studie jedoch z.B. fragt, wie Personen Sinn aus Gewalterfahrungen generieren, welche Narrative gewaltbereite Personen ex post heranziehen, um Gewalthandlungen zu rechtfertigen oder in welchem Umfeld gewaltaffine Personen aufwachsen, sind Interviews und teilnehmende Beobachtung besser geeignete methodische Ansätze. Wer die Fragestellung untersuchen möchte, wie viele Personen den Eindruck haben, Gewalt nehme zu und wie dies ihr Wahlverhalten beeinflusst, sind Survey-Umfragen der geeignetere Weg. Wenn Forschende verstehen möchten, welche Eigenschaften des Wohnumfelds zu höheren Gewaltraten führen, sind offizielle Statistiken zu Gewalttaten und urbanen Räumen besonders hilfreich. VDA eignet sich also nicht für jede Fragestellung der Gewaltforschung. Für die Analyse dessen, was in Gewaltsituationen tatsächlich passiert, wie sie entstehen, ablaufen und enden, bieten neuere Videodaten und VDA zugleich entscheidende neue Einblicke und ungeahnte Möglichkeiten.

Selbst für Studien mit diesem Fokus stoßen Videodaten teils an ihre Grenzen. Z.B. sind Forschende oft nicht nur daran interessiert, eine Gewaltsituation zu untersuchen, sondern wollen auch den Einfluss situativer Dynamiken zur Gewalt mit dem Einfluss von Kontextfaktoren vergleichen oder interessieren sich zusätzlich für die Interpretationen der Situation durch Anwesende. Hier zeigt sich das große 
Potenzial von Triangulation und Mixed Methods-Ansätzen. VDA betont daher dezidiert die Vorteile, Videodaten mit Dokumentdaten, teilnehmender Beobachtung und Interviews zu verbinden.

\section{Triangulation und Mixed Methods in der Gewaltforschung}

Mixed Methods gewinnen seit Anfang der 2000er Jahre zunehmend an Bedeutung, international wie auch im deutschsprachigen Raum (Creswell und Plano 2017; Kuckartz 2014, S. 7). Triangulation meint die Kombination verschiedener Datentypen und Erhebungsverfahren (Flick 1992); Mixed Methods-Ansätze meint die Verbindung qualitativer und quantitativer Analyseansätze (Creswell und Plano 2017). Das Ziel ist in beiden Fällen, komplementäre Stärken zu nutzen und mögliche Schwächen einzelner Datentypen oder Analysemethoden auszugleichen. Dieses Ziel macht Triangulation und Mixed Methods-Ansätze für die Gewaltforschung enorm wertvoll. Erstens können so mögliche Verzerrungen im Datenmaterial ausgeglichen werden, indem verschiedene Datenquellen oder Datentypen kombiniert werden. Zweitens können blinde Flecken von Videodaten beleuchtet werden: Viele Studien, die neuere Videodaten nutzen, um situative Dynamiken zu erforschen (z. B. Collins 2008; Klusemann 2009; Nassauer 2018b, 2019b), möchten z. B. auch Kontextfaktoren wie Marginalisierung, Deprivation oder Gewaltmotivationen als alternative oder ergänzende Erklärungen überprüfen oder die Interpretationen der beteiligten Personen in der Situation in die Analyse einbeziehen.

Erstens kann Triangulation verschiedene Videos betreffen, z. B. durch die Nutzung mehrerer Videos zum selben Ereignis oder von Videos, die von verschiedenen Gruppen produziert wurden. Dies kann bei der optimalen Erfassung der Situation helfen und ist besonders relevant, wenn Forschende an situativen Abläufen vor einer Gewalthandlung interessiert sind, filmende Personen aber bestimmten Interessengruppen angehören oder anderweitig voreingenommen sind. Z. B. trianguliert Nassauer (2016, 2018b, 2019b) Videodaten für ihre Analyse von Interaktions- und Emotionsdynamiken vor der Entstehung von Protestgewalt. Sie nutzt zu vielen Situationen innerhalb der Proteste mehrere Videoaufnahmen und verwendet Daten verschiedenster Quellen (Polizei, Demonstrierende, Medien, andere Beobachter) um sicherzustellen, dass keine der Parteien bestimmte Momente oder Handlungen im Protestverlauf bewusst ausgelassen hat (z. B. durch Schnitt oder Auswahl des Bildausschnitts).

Eine zweite Form der Triangulation kombiniert verschiedene Datentypen, wie z. B. Videodaten mit Dokumenten, Interviewdaten, teilnehmende Beobachtung oder Big Data (z.B. Twitter-Beiträge, siehe unten). Dies kann wichtig sein, wenn Forschende an der Interpretation der Situation durch Akteur*innen oder an der Rolle von Kontextfaktoren in der Gewaltentstehung interessiert sind. Letzteres ist gerade im Licht der aktuellen Debatte um Mikro- vs. Makro-Faktoren für Gewaltentstehungen wichtig (Collins 2019b; u.a. Hoebel und Malthaner 2019; Knöbl 2019; Nassauer 2019b): Forschende können etwa zunächst durch Interviews oder Dokumente Kontextfaktoren zur Gewalt untersuchen und in einem nächsten Schritt durch Videodaten, teilnehmende Beobachtung und Dokumente zu situativen Ab- 
läufen die Rolle der Mikro-Ebene erfassen. Eine Reihe von Studien wählt diesen Weg. Bspw. nutzt Klusemann (2009) in seiner Analyse des Massakers von Srebrenica Videomaterial und Dokumente. Anhand einer achtstündigen Videoaufzeichung analysiert er Körpersprache, Blickkontakt und Emotionsausdrücke sowie die Konversation in Verhandlungen zwischen dem serbischen General Mladić und UNKommandant Karremans am Vortag des Massakers. Gleichzeitig analysiert Klusemann Dokumente, um die Rolle von Hintergrundfaktoren, wie ethnischen Hass, für den Ausbruch des Massakers zu prüfen. Mit einem ähnlichen Vorgehen erhebt Nassauer (2016, 2018b, 2019b) in ihrer Studie zu Protestgewalt neben Videodaten verschiedener Quellen auch Dokumentdaten, wie Polizeiberichte, Berichte von Demonstrierenden, Medienberichte und Berichte anderer Beobachter*innen. Ziel ist zum einen, die situativen Abläufe während der Demonstration zu verifizieren und zum anderen ermöglicht das Vorgehen, Informationen zu Kontextfaktoren zu erheben (z.B. zur vorherigen Polizeistrategie, Polizei- und Protestkultur, Gruppe und Motivationen der Demonstrierenden, Verhältnis von Polizei zu Demonstrierenden oder Gewalterwartungen). Zusätzlich trianguliert Nassauer diese Daten mit Interviews und teilnehmender Beobachtung, um weitere Informationen zu Polizei- und Protestkultur sowie zu Kontextfaktoren zu gewinnen, um so den Einfluss von Kontext und situativen Dynamiken zur Gewalt zu vergleichen und ihre Wechselwirkung zu untersuchen. Beide Studien kommen zu dem Schluss, dass situative Dynamiken maßgeblich für Gewaltausbrüche sind und Kontextfaktoren nicht systematisch erklären können wo, wann oder warum Gewalt entsteht (für ähnliche Ergebnisse aus einer Studie zu Protesten während des Arabischen Frühlings siehe Bramsen 2018).

Als eine dritte Form der Triangulation können Gewaltforschende Verfahren der Datenerhebung kombinieren. So kann bspw. zunächst ein theoretisches Sampling verwendet werden, um Konzepte aus den Daten (Videodaten, Interviews, teilnehmende Beobachtung oder Dokumente) zu entwickeln, um die erarbeiteten Zusammenhänge dann anhand zufällig ausgewählter Fälle zu testen. Nassauer (2019b) entwickelt bspw. anhand von vier Fällen Konzepte zu Interaktionen, die zur Gewalt in Protesten führen können. In einem zweiten Schritt zieht sie dann ein Zufallssample von weiteren 26 friedlichen und gewaltsamen Protesten in Deutschland und den USA aus zwei Datensätzen (Stanford University 2009; Wissenschaftszentrum Berlin für Sozialforschung 2011), um die Relevanz der erarbeiteten Konzepte in diesem Sample zu untersuchen. Ein solches Forschungsdesign unterzieht die erarbeiteten Konzepte und Muster einem vergleichsweise harten Test der Reichweite. Ähnlich könnten Studien auch selbstgefilmte Videos eines Ereignisses mit Online-Videos desselben Typs von Ereignis kombinieren, um durch diesen Mix von Strategien der Datenerhebung die Stärken beider Ansätze zu bündeln. Eine weitere Möglichkeit besteht in der Einbettung von Anwendungen der Virtuellen Realität zusammen mit etablierten Forschungsmethoden (Drury et al. 2009; Hess 2019). Eine solche Triangulation von Datentypen und Datenerhebung verspricht für die Gewaltforschung enorme Vorteile, da speziell das Phänomen der Gewalt, wie oben besprochen, häufig verzerrt wahrgenommen wird und einzelne Quellen möglicherweise Aufmerksamkeit erregen, Rechtfertigungen bereitstellen oder Anschuldigungen anbringen wollen (Heitmeyer und Hagan 2002; Vrij et al. 2014; Nassauer 2019a). 
Schließlich können Gewaltstudien davon profitieren, Mixed Methods anzuwenden und verschiedene Analyseansätze zu kombinieren. Z.B. können in einem sequentiellen Forschungsdesign (Small 2011, S. 67) VDA und Grounded Theory in einer ersten Analyse Konzepte entwickeln, um dann anhand dieser Konzepte mithilfe von Qualitative Comparative Analysis (QCA, siehe Ragin 2008; Legewie 2013) oder Sequence Analysis (Abbott et al. 2004; Aisenbrey und Fasang 2010) in systematischer und formalisierter Weise nach Mustern zu suchen. Ein solcher Ansatz wurde bspw. von Nassauer (2019b) in ihrer Studie zu Protestgewalt gewählt. ${ }^{4}$ Eine weitere Möglichkeit ist, Gewaltausbrüche zunächst qualitativ zu untersuchen (z.B. Liebst et al. 2019) und dann durch Regressionsanalyse und Regressionsbäume zu erforschen, welche Faktoren systematisch zur Gewalt in einem größeren Sample führen (bspw. ähnliche Mikro-Faktoren kodierend, siehe Levine et al. 2011).

Triangulation und Mixed Methods-Studien werden voraussichtlich in der Zukunft weiter an Relevanz gewinnen. Sie können die Reliabilität und Validität von Studien erhöhen (Small 2011) und es der Gewaltforschung ermöglichen, Kontext- und situative Faktoren der Gewalt systematisch und nachvollziehbar zu untersuchen. Sie können dabei helfen, dichte und detaillierte Konzepte zur Gewaltentstehung zu erarbeiten und deren Relevanz über eine Bandbreite gewaltsamer Ereignisse zu erforschen. In Zukunft könnten besonders Mixed Methods-Ansätze fruchtbar und erfolgsversprechend sein, um etablierte und neuere Methoden der Gewaltforschung mit Big Data und Computational Social Sciences zu verknüpfen.

\section{Big Data und Computational Social Sciences}

Big Data und so genannte Computational Social Sciences, die häufig digitale Datenbestände nutzen und auf Techniken der Informatik und Data Science zurückgreifen, haben zweifelsfrei Konjunktur. Wie immer ist damit auch ein gewisses Maß an überzogenen Erwartungen verbunden (siehe etwa das Scheitern von Googles Algorithmus zur Voraussage von Grippewellen, diskutiert in Lazer et al. 2014). Dennoch haben Big Data und Computational Social Sciences der sozialwissenschaftlichen Forschung enorme Potenziale eröffnet und schon jetzt wichtige Beiträge geleistet, vor allem, wenn sie mit ,traditionellen“ Methoden verknüpft werden (für einen hervorragenden Überblick siehe Salganik 2018). Im folgenden Abschnitt werden beispielhaft drei Bereiche vorgestellt, in denen Big Data und Computational Social Science zur Gewaltforschung beitragen können. Erstens können Web Crawling und Web Scraping die Datenerhebung unterstützen und teilweise automatisieren. Zweitens bietet die computergestützte Textanalyse neue Möglichkeiten, Kontextfaktoren zur Gewalt zu erforschen. Drittens gibt es neuere Entwicklungen im Feld der Coтриter Vision, besonders im Bereich der Human Action Recognition, die automatisierte Analysen von Videodaten ermöglichen.

\footnotetext{
${ }^{4}$ Eine solche Methodentriangulation kann ebenso unter Verwendung ethnographischer oder anderer Datentypen vorgenommen werden.
} 


\subsection{Automatisierung der Datenerhebung durch Web Crawling und Web Scraping}

Web Crawling und Scraping bieten zeiteffiziente Lösungen für Datenerhebungsschritte, die zwar nicht hochkomplex sind, aber häufig wiederholt werden müssen. Web Crawling bedeutet, dass ein Suchalgorithmus (Bot) im Internet nach bestimmten Inhalten sucht, ähnlich den gängigen Suchmaschinen. Web Scraping bedeutet, ausgewählte Daten aus einem Web Crawl automatisch zu kopieren und zu speichern. Für die Gewaltforschung bieten sie eine Reihe spannender Anwendungsmöglichkeiten. Wir stellen hier zwei mögliche Anwendungen im Kontext der video-basierten Gewaltforschung vor: Erstellen von Samples und ergänzende Erhebung von Daten (für eine ausführlichere Beschreibung, siehe Legewie 2019; Legewie et al. 2019, S. $22 \mathrm{ff}$.).

Web Crawling und Scraping können Forschenden bspw. beim systematischen Erstellen eines Samples helfen, wenn Videodaten online gesammelt werden. Z. B. ist eine der ersten Fragen bei der Verwendung von Online-Videodaten zur Untersuchung von Gewaltereignissen, welche Fälle ausgewählt werden sollten und wie der Datensatz oder das Sample reproduzierbar erstellt werden kann. Für quantitative Untersuchungen ist das systematische Erstellen eines Samples unabdingbar (in diesem Fall auf Basis einer Nicht-Zufallsstichprobe; siehe hierzu Baker et al. 2013). Für explorative Studien mit geringen Fallzahlen ist eine schrittweise, theoriegeleitete Fallauswahl zwar möglich, aber langwierig und schwer reproduzierbar (siehe Nassauer 2018a). Darüber hinaus hat es für explorative Studien mit kleiner Fallzahl Vorteile, zunächst ein großes Sample zu erstellen, um eine bessere Übersicht über die Fälle zu bieten, eine zielgerichtetere Fallauswahl zu ermöglichen und die Dokumentation des Prozesses zu erleichtern. Web Crawling und Web Scraping können eine Liste von Suchbegriffen systematisch anwenden, um bspw. eine Auswahl relevanter Videos zusammenzustellen. Die Bereitstellung des Programmcodes der Anwendung kann dann das Vorgehen für andere Forschende reproduzierbar machen. Die Auswahl kann bereits auf bestimmte Videos enggeführt werden, wie etwa auf bestimmte Uploader*innen oder Arten von Uploader*innen (z. B. Polizei, NGOs, Nachrichtenagenturen). Dies kann Forschenden helfen, nur zuverlässige Quellen zu untersuchen oder ausgewogene Arten von Quellen einzubeziehen (siehe oben). Zudem können Meta-Daten automatisiert gesammelt werden, wie z.B. die Anzahl der Klicks, die Länge des Videos, die maximal verfügbare Auflösung, der/die Name/ $n$ der/des Uploader*in und Kontaktlinks, die Anzahl der Kommentare etc. Durch die Gesamtzahl der Videos im (vorläufigen) Sample sowie durch die genannten Metadaten können Forschende, die eine qualitative oder quantitative Fallauswahl reproduzieren wollen, zudem das Ausmaß des „Drifts“ der genutzten Plattform einschätzen (Salganik 2018, S. 33). Drift bezeichnet Veränderungen in den Nutzern oder dem Suchalgorithmus einer Plattform, was zu veränderten Suchergebnissen führen kann. Da Forschende auf Basis des Programmcodes die Suchanfrage durch denselben Bot durchführen können, kann das resultierende Sample mit dem ursprünglichen Sample verglichen werden, um das Ausmaß der Abweichung zu bewerten. So kann die Sampleauswahl einer Gewaltstudie unter Verwendung von Videodatenanalyse nachvollzogen oder reproduziert werden. Ein ähnlicher Ansatz 
kann für die Sampleauswahl in Studien mit anderen Online-Daten, wie etwa Nachrichtenartikeln, angewandt werden (Gong 2011).

Webcrawling und Webscraping können ferner zur Erhebung von nicht-visuellen Onlinedaten genutzt werden. Solche zusätzlichen Daten spielen in der Gewaltforschung oft eine Rolle, wenn Ereignisse aus dem realen Leben untersucht werden. Sie können für Gewaltforschende bspw. relevant sein, um zusätzliche Informationen zu einem Gewaltereignis zu erhalten, bei dem eine teilnehmende Beobachtung oder Interviews durchgeführt wurden. Web Crawling und Scraping können es Gewaltforschenden so ermöglichen, umfassendere und verlässlichere Erkenntnisse über ein Gewaltereignis zu erlagen, als dies bisher der Fall war. Ebenso können sie Informationen ergänzen, die im Video selbst nicht sichtbar sind, z. B. zu Interpretationen, Kontexten und Kommunikationen zwischen Akteur*innen in großen Gruppen. Bislang haben Forschende solche Daten manuell erhoben (z. B. Nassauer 2018a, b), was zwar möglich, aber enorm zeitaufwendig ist. Web Crawling und Scraping können einige dieser Aufgaben automatisieren. Budak und Watts (2015) schlagen etwa einen Ansatz zur Identifizierung und Analyse von Twitter-Nutzer*innen vor, die bei den Gezi-Protesten in der Türkei im Jahr 2013 teilnahmen. Solche Daten können dazu beitragen, Interpretationen der Teilnehmenden von Ereignissen während des Protests zu untersuchen und helfen zu verstehen, wie sich Informationen zwischen Teilnehmenden während eines Protests verbreiten. Es ist nach wie vor relativ einfach, Twitter-Kommentare während zeitgleich ablaufender Ereignisse zu speichern, die ein*e Forscher*in mit Videos analysieren möchte. ${ }^{5}$ In ähnlicher Weise können Forschende Kommentare aus Livestreams oder Online-Videos per Scraping speichern, um zu untersuchen, wie Nutzer*innen auf ein bestimmtes Thema reagieren oder um zusätzliche Informationen zu einem bestimmten Ereignis zu finden.

Eine weitere Möglichkeit für den Einsatz von Web Crawling und Scraping zur Erhebung zusätzlicher Daten ist die Suche nach ergänzenden Informationen, die auf mehreren verschiedenen Webseiten und Plattformen zugänglich sind (Legewie 2019), wie etwa Medien-, Polizei- und Augenzeugenberichte. Z.B. schlägt Gong (2011) einen Ansatz vor, um ein Snowball Sample von Webseiten zu erstellen. Der Ansatz könnte verwendet werden, um automatisch eine Liste mit Webseiten $\mathrm{zu}$ erstellen, die relevante Informationen zu einem bestimmten Ereignis enthalten (z.B. ein gewaltsamer Protest, eine Massenschießerei, ein terroristischer Anschlag). Mithilfe eines zusätzlichen, für das entsprechende Projekt erstellten Themenfilters könnte dieses automatisierte Sample zusätzlich präzisiert werden und würde auf Basis des gesamten Textes der Webseite anstelle einzelner Stichworte nach relevanten Webseiten suchen, was eine zugleich präzisere und reichhaltigere Datenerhebung verspricht (Bail 2014, S. 10).

Für Videodatenforscher*innen kann der Mangel an technischen Fähigkeiten zur Programmierung von Web Crawling und Scraping Bots eine Herausforderung darstellen. Eine Lösung für diese Herausforderung könnte das von Chasins entwickelte Helena Web Scraping Tool sein. Es bietet eine barrierefreie Plattform zum Erstel-

\footnotetext{
5 Der Zugriff auf ältere Twitter-Daten erfordert mittlerweile finanzielle Ressourcen, bei entsprechenden Forschungsvorhaben sollten also die nötigen Mittel im Antrag berücksichtigt werden.
} 
len von Web Scraping Anwendungen, die keine technischen Kenntnisse erfordert (Barman et al. 2016; Chasins et al. 2015; siehe auch http://helena-lang.org).

\subsection{Computergestützte Textanalyse zur Erforschung von Kontextfaktoren}

Computergestützte Textanalyse hat seit der Jahrtausendwende rasante Fortschritte erzielt und findet in den Sozialwissenschaften immer breitere Anwendung (siehe u. a. Eichstaedt et al. 2015; Franzosi 2012; McFarland et al. 2013; Merz et al. 2016; für eine Übersicht methodischer Ansätze siehe Schwartz und Ungar 2015). Für die Gewaltforschung bietet die computergestützte Textanalyse mannigfaltige Anwendungsmöglichkeiten. Als Beispiel sei hier die Erforschung von Kontextfaktoren mithilfe von geo-identifizierten Twitter-Daten genannt.

Twitter-Daten bieten einen reichhaltigen Korpus für menschliche Sprache, aus der sich eine Reihe relevanter Informationen gewinnen lassen. So haben Studien u.a. Parteineigung (Budak und Watts 2015) und Persönlichkeit (Schwartz et al. 2013) aus individuellen Twitter-Daten identifiziert, die Prävalenz von Herzkrankheiten in bestimmten Regionen durch aggregierte Twitter-Daten erklärt (Eichstaedt et al. 2015) oder Twitter-Posts dafür genutzt, die Ursachen für das Sympathisieren mit der terroristischen Vereinigung Islamischer Staat (ISIS) zu untersuchen (Magdy et al. 2015). Ähnliche Techniken könnten in der Gewaltforschung eingesetzt werden, um z.B. einen möglichen Zusammenhang zwischen Gewalttaten und latentem Aggressionsniveau (operationalisiert als Anteile aggressiver Sprache auf Twitter) oder wahrgenommener Benachteiligung (operationalisiert als Anteil der Tweets, die erlebte Benachteiligung ausdrücken) über regionale Einheiten hinweg zu untersuchen. Studien könnten dazu längsschnittliche, lokal verortete Twitter-Daten mit längsschnittlichen Survey-Daten verknüpfen und untersuchen, ob ein Anstieg des Aggressionsniveaus oder der wahrgenommenen Benachteiligung auf Twitter zu einem Anstieg tatsächlicher Gewalttaten führt. Solche Analysen könnten wichtige Beiträge zur Debatte um Kontext- und situative Faktoren in der Gewaltentstehung liefern.

\subsection{Videodatenanalyse und Human Action Recognition}

Human Action Recognition bezeichnet computergestütztes Kodieren von Handlungen und Interaktionen in Videoaufnahmen (Sigurdsson et al. 2016, 2017; Yeung et al. 2015; für eine Einführung siehe Legewie et al. 2019, S. 16ff..; Russakovsky 2019). Human Action Recognition ist ein überaus dynamisches Feld, das schon bald enorme weitere Fortschritte erzielt haben und für die video-basierte Gewaltforschung weitere Potenziale bieten wird. Bereits heute könnten Techniken der Human Action Recognition in der Gewaltforschung eingesetzt werden, um z. B. automatisch zu kodieren, wann Menschen in Richtung der Kamera blicken (z.B. um Reaktivität zu überprüfen, siehe oben), um grobe Unterscheidungen in der Körperhaltung automatisch zu erfassen (z. B. um Konzepte wie Collins' (1993, 2008) emotionale Energie zu operationalisieren) oder um die Distanz zwischen Akteur*innen automatisch erfassen zu lassen (z.B. um die Verletzung persönlichen Raums in einer Interaktion zu erfassen; für diese und weitere Beispiele siehe Legewie et al. 2019: $13 \mathrm{ff}$.$) .$ 
Diese Techniken könnten Forschende nutzen, um größere Video-Datensätze von Gewalthandlungen maschinell zu kodieren und so weitere Antworten zum Einfluss situativer Prozesse und Dynamiken auf die Gewaltentstehung zu finden. Ein Anwendungsbeispiel wäre erneut der Datensatz an Body-Camera-Videos des LAPD (McCluskey et al. 2019; Uchida 2019). Aber auch Betreiber*innen des öffentlichen Nahverkehrs, Städte und Sicherheitsfirmen verfügen in vielen Großstädten ebenfalls über Millionen von Videos. Selbst wenn sich Forschende auf einen sehr spezifischen Aspekt in den Interaktionen vor dem Gewaltausbruch konzentrieren würden (z. B. „Bewegte sich das Opfer vom/von der Täter*in weg oder auf den/diese zu, bevor diese*r Gewalt ausübte?“ oder ,Zeigte der/die Täter*in Aggressivität vor der Gewalthandlung?"), wäre es schier unmöglich, diese Millionen von Videos in einem zeitlich angemessenen Rahmen zu analysieren. Forschende können diese Daten in der Zukunft in Mixed Methods-Studien nutzen, um bspw. zunächst an einem kleineren Sample bestimmte potenziell relevante Aspekte zu identifizieren und eine begrenzte Anzahl von Videos zu kodieren. Diese können dann an größeren Datensätzen mithilfe etablierter Algorithmen untersucht werden (eine aktuelles Beispiel eines solchen Algorithmusses ist etwa Googles BERT, siehe Devlin et al. 2019). Solche Algorithmen würden an öffentlichen Datensätzen zunächst darauf trainiert (pretrained) Handlungen erfassen zu können, würden dann anhand eines Subsamples der kodierten Videos auf den speziellen Anwendungsfall trainiert, um dann auf das gesamte Sample angewendet zu werden. Zur Reliabilitätsprüfung würde aus dem Anwendungssample wiederum ein zufällig gezogener Teil der Videos von Hand kodiert werden. Dieses Anwendungsbeispiel stellt nur eines von vielen möglichen Designs dar, um video-basierte Gewaltforschung mit Human Action Recognition zu verbinden (Legewie et al. 2019, S. 16ff.).

Human Action Recognition kann zudem genutzt werden, um einen großen Datensatz auf relevante Interaktionen zu sichten. Wenn Forschende bspw. Millionen von Videos gegenüberstehen, wäre ein Durchgehen von 10 Mio. Videos per Hand schier unmöglich, um relevante Videos zu identifizieren. Human Action Recognition könnte helfen, unter all diesen Videos jene zu identifizieren, in denen Gewalt ausbrach (z.B. operationalisiert als ,eine Person wird von einer anderen Person geschlagen oder getreten“). Algorithmen der Human Action Recognition könnten, gefüttert mit ausreichend Daten als „Lernmaterial“, ebenso all jene Videos filtern, in denen Personen einander geschubst, aber daraufhin nicht gewalttätig gehandelt haben. Eine solche Vorauswahl kann es Forschenden dann erlauben, die begrenzte Anzahl der Videos genau zu analysieren, die diesen Kriterien entsprechen.

Human Action Recognition ist ein sich rasant entwickelndes Feld, das speziell Gewaltforschenden angesichts des internationalen Booms situativer Ansätze (Collins 2019b) helfen kann, solche Theorien quantitativ, anhand großer Datensätze zu untersuchen und so die bisherigen Ergebnisse detaillierter Fallanalysen von Gewaltereignissen fruchtbar zu ergänzen und weiter auszubauen. 


\section{Zusammenfassung und Ausblick}

Die Gewaltforschung kann enorm von jüngeren Entwicklungen sozialwissenschaftlicher Methoden profitieren. Seit der Jahrhundertwende stehen Forschenden eine Reihe neuer Ansätze zur Verfügung: Es gibt immer mehr Videoaufnahmen von gewaltsamen Ereignissen, Triangulation und Mixed Methods-Methoden werden stetig weiterentwickelt und durch Computational Social Sciences finden Big Data-Ansätze Einzug in immer mehr Forschungsfelder. Der vorliegende Beitrag nahm eine methodologische Bestandsaufnahme vor und diskutierte den gegenwärtigen und zukünftigen Einfluss von Videodatenanalyse, Triangulation und Mixed Methods-Ansätzen und Big Data auf die quantitative und qualitative Gewaltforschung.

Neuere Videodaten und methodische Entwicklungen in der Videodatenanalyse bieten vielversprechende Einblicke in Gewalthandlungen und ermöglichen neue Perspektiven auf Gewaltentstehung. Sie ermöglichen es, transparent und systematisch zu erforschen, was vor und während einer Gewalthandlung passierte, wie Gewalt ablief und wie sie endete. Dank dieser Einblicke können Forschende Muster in situativen Dynamiken untersuchen und neue Einblicke zur Relevanz situativer Faktoren für die Gewaltentstehung liefern. Im Beitrag stellten wir die Videodatenanalyse (VDA) als einen möglichen methodischen Ansatz zur Analyse neuerer Videodaten vor. Ziel von VDA ist es, einen interdisziplinären methodologischen Rahmen zu bieten, hilfreiche Perspektiven und Werkzeuge zur Analyse neuerer Videodaten zu liefern und den interdisziplinären Austausch zur situativen Analyse von Gewalt zu fördern. VDA ist jedoch nicht für jede Fragestellung sinnvoll: Zur Erforschung von ex-post Rechtfertigungen oder Lebensumständen gewaltbereiter Personen, zur Untersuchung zur Darstellung von Gewalt in Videos und anderen Medien oder zur Analyse von Einstellungen zur Gewalt können andere Datentypen oder andere Ansätze der Videoanalyse Einsicht geben.

Triangulation und Mixed Methods-Studien erlauben es, die Reliabilität und Validität von Studien zu erhöhen (Small 2011) und ermöglichen es, situative und Kontextfaktoren der Gewalt systematisch und nachvollziehbar zu untersuchen. Forschende können u. a. verschiedene Videodaten triangulieren oder Videodaten und Dokumentdaten kombinieren (beides in der Videodatenanalyse und situativen Gewaltanalyse häufig verwendete Ansätze). Sie können aber auch verschiedene Erhebungsverfahren und Analyseansätze verknüpfen.

Zudem können Big Data und Computational Social Sciences bestehende Ansätze zur Erforschung von Gewalt bereichern und gänzlich neue Ansätze hervorbringen. Web Crawling und Scraping können Arbeitsschritte in der Datenerhebung automatisieren. Computergestützte Textanalysen, wie etwa die Analyse von Twitter-Daten, können u.a. Beiträge zur Diskussion um Kontext- und situative Faktoren in der Gewaltentstehung liefern. Und automatisierte Videoanalysen durch Human Action Recognition können die Videodatenanalyse erleichtern und langfristig Videodaten als Big Data zugänglich machen.

Die skizzierten Entwicklungen bergen zugleich methodologische Herausforderungen, von denen hier nur vier genannt werden sollen: Erstens wird Software, die das Fälschen von Videodaten ermöglicht, stetig weiterentwickelt. Je verbreiteter gefälschte Videodaten sind, desto mehr stellt dies die Videodatenanalyse in der 
Zukunft vor Schwierigkeiten, sofern Software zur Erkennung solcher Fälschungen nicht Schritt hält (siehe z. B. Guera und Delp 2018 für einen aktuellen Ansatz). Zweitens kann die situative Gewaltforschung und die Verwendung von Videodaten zur Analyse sozialer Phänomene Forschende vor immer neue ethische Probleme stellen. Ein Beispiel ist hier die Verwendung von Livestreams terroristischer Anschläge wie in Christchurch (März 2019) oder Halle (Oktober 2019; siehe Legewie und Nassauer 2020). Im Bereich von Big Data ist dagegen die De-Anonymisierung und die große Reichweite möglicher Datenleaks ein Kernthema der Forschungsethik (Salganik 2018, S. 281 ff.). Diese Entwicklungen erfordern stetige Reflektion über die ethische Verwendbarkeit von Daten in der Gewaltforschung und darüber hinaus sowie eine aktive Forschungsgemeinschaft, die gemeinsam an Richtlinien und neuen Lösungen arbeitet. Eine Schwierigkeit im Bereich der Computational Social Sciences in der Gewaltforschung ergibt sich aus den oft eingeschränkten technischen Fähigkeiten, bspw. Human Action Recognition oder Web Scraping in Studien einzusetzen. Hier können Kooperationen mit Informatiker*innen und anderen Forschenden, oder auch nutzungsfreundliche Angebote, wie das Helena Web Scraping Tool, hilfreich sein. Viertens liegt eine Herausforderung im Bereich von Big Data darin, sachlich nach sinnvollen Anwendungen zu suchen und solche Ansätze weder unkritisch einzusetzen noch kategorisch abzulehnen. Neben Big Data Anwendungen werden weiterhin klassische Survey-Forschungen und robuste, analytisch-empirische qualitative Forschungen erforderlich sein, die die Mechanismen, Dynamiken und Prozesse der Gewalt untersuchen. Ebenso bedarf es fundierter Theorien und Überlegungen, die die Gewaltforschung auf eine starke theoretische Basis stellen, die wir empirisch überprüfen können. Eine Verknüpfung etablierter und neuer Methoden verspricht daher eine fruchtbare Zukunft für die Gewaltforschung.

Funding Open Access funding provided by Projekt DEAL.

Open Access Dieser Artikel wird unter der Creative Commons Namensnennung 4.0 International Lizenz veröffentlicht, welche die Nutzung, Vervielfältigung, Bearbeitung, Verbreitung und Wiedergabe in jeglichem Medium und Format erlaubt, sofern Sie den/die ursprünglichen Autor(en) und die Quelle ordnungsgemäß nennen, einen Link zur Creative Commons Lizenz beifügen und angeben, ob Änderungen vorgenommen wurden.

Die in diesem Artikel enthaltenen Bilder und sonstiges Drittmaterial unterliegen ebenfalls der genannten Creative Commons Lizenz, sofern sich aus der Abbildungslegende nichts anderes ergibt. Sofern das betreffende Material nicht unter der genannten Creative Commons Lizenz steht und die betreffende Handlung nicht nach gesetzlichen Vorschriften erlaubt ist, ist für die oben aufgeführten Weiterverwendungen des Materials die Einwilligung des jeweiligen Rechteinhabers einzuholen.

Weitere Details zur Lizenz entnehmen Sie bitte der Lizenzinformation auf http://creativecommons.org/ licenses/by/4.0/deed.de.

\section{Literatur}

Abbott, Andrew Delano, und Heather MacIndoe. 2004. Sequence analysis and optimal matching techniques for social science data. In Handbook of data analysis, Hrsg. Melissa Hardy, Alan Bryman, 387-405. London: SAGE.

Aisenbrey, Silke, und Anette E. Fasang. 2010. New life for old ideas: the "second wave" of sequence analysis bringing the "course" back into the life course. Sociological Methods \& Research 38:420-462. 
ASA. 2018. Code of ethics of the ASA committee on professional ethics. American Sociological Association (ASA). https://www.asanet.org/sites/default/files/asa_code_of_ethics-june2018a.pdf

Athens, Lonnie H. 1980. Violent criminal acts and actors: a symbolic Interactionist study. Boston: Routledge \& Kegan Paul.

Atkinson, Paul Anthony, Sara Delamont, Amanda Coffey, John Lofland, und Lyn H. Lofland. 2007. Handbook of ethnography. Los Angeles: SAGE.

Bail, Christopher A. 2014. The cultural environment: measuring culture with big data. Theory and Society 43:465-482.

Baker, Reg, J. Michael Brick, Nancy A. Bates, Mike Battaglia, Mick P. Couper, Jill A. Dever, Krista J. Gile, und Roger Tourangeau. 2013. Summary report of the AAPOR task force on non-probability sampling. Journal of Survey Statistics and Methodology 1:90-143.

Barman, Shaon, Sarah Chasins, Rastislav Bodik, und Sumit Gulwani. 2016. Ringer: web automation by demonstration. In Proceedings of the 2016 ACM SIGPLAN international conference on object-oriented programming, systems, languages, and applications. OOPSLA 2016., 748-764. New York: ACM.

Bernard, H. Russell, Peter Killworth, David Kronenfeld, und Lee Sailer. 1984. The problem of informant accuracy: the validity of retrospective data. Annual Review of Anthropology 13:495-517.

Bramsen, Isabel. 2018. How violence happens (or not): situational conditions of violence and nonviolence in Bahrain, Tunisia and Syria. Psychology of Violence 8:305-315.

BSA. 2017a. Ethics guidelines and collated resources for digital research. Statement of ethical practice Annexe. British Sociological Association (BSA). https://www.britsoc.co.uk/media/24309/bsa_ statement_of_ethical_practice_annexe.pdf. Zugegriffen: 18. Febr. 2020.

BSA. 2017b. Statement of ethical practice. British Sociological Association (BSA). https://www.britsoc. co.uk/media/24310/bsa_statement_of_ethical_practice.pdf. Zugegriffen: 18. Febr. 2020.

Budak, Ceren, und Duncan J. Watts. 2015. Dissecting the spirit of Gezi: influence vs. Selection in the occupy Gezi movement. Sociological Science 2:370-397.

Campbell, John L., Charles Quincy, Jordan Osserman, und Ove K. Pedersen. 2013. Coding in-depth semistructured interviews problems of Unitization and Intercoder reliability and agreement. Sociological Methods \& Research 42:294-320.

Chasins, Sarah, Shaon Barman, Rastislav Bodik, und Sumit Gulwani. 2015. Browser record and replay as a building block for end-user web automation tools. In Proceedings of the 24th international conference on World Wide Web, 179-182. WWW'15 Companion. New York: ACM.

Collins, Randall. 1993. Emotional energy as the common denominator of rational action. Rationality and Society 5:203-230.

Collins, Randall. 2008. Violence: a micro-sociological theory. Princeton: Princeton University Press.

Collins, Randall. 2019a. "The visual data revolution in social science". Vortrag, Blankensee-Colloquium on Capturing and Analyzing Social Change, Berlin.

Collins, Randall. 2019b. Preventing violence: insights from micro-sociology. Contemporary Sociology 48:487-494.

Creswell, John W., und Vicki L.Clark Plano. 2017. Designing and conducting mixed methods research, 3. Aufl., Los Angeles: SAGE.

Devlin, Jacob, Ming-Wei Chang, Kenton Lee, und Kristina Toutanova. 2019. BERT: pre-training of deep bidirectional transformers for language understanding. arXiv:1810.04805 [cs].

DGS, und BDS. 2017. Ethik-Kodex der Deutschen Gesellschaft für Soziologie (DGS) und des Berufsverbandes Deutscher Soziologinnen und Soziologen (BDS). Deutsche Gesellschaft für Soziologie, Bund Deutscher Soziologinnen und Soziologen

Dingwall, Robert. 2019. 21st century methods and 20th century thinking. Vortrag, Blankensee-Colloquium on Capturing and Analyzing Social Change, Berlin.

Drury, John, Chris Cocking, Steve Reicher, Andy Burton, Damian Schofield, Andrew Hardwick, Danielle Graham, und Paul Langston. 2009. Cooperation versus competition in a mass emergency evacuation: a new laboratory simulation and a new theoretical model. Behavior Research Methods 41:957-970.

Eichstaedt, Johannes C., Hansen Andrew Schwartz, Margaret L. Kern, Gregory Park, Darwin R. Labarthe, Raina M. Merchant, Sneha Jha, Megha Agrawal, Lukasz A. Dziurzynski, Maarten Sap, Christopher Weeg, Emily E. Larson, Lyle H. Ungar, und Martin E.P. Seligman. 2015. Psychological language on twitter predicts county-level heart disease mortality. Psychological Science 26:159-169.

Elsner, Claudia, und Annie E. Wertz. 2019. The seeds of social learning: Infants exhibit more social looking for plants than other object types. Cognition 183:244-255.

Erickson, Frederick. 2011. Uses of video in social research: a brief history. International Journal of Social Research Methodology 14:179-189. 
Felson, Richard, und Henry J. Steadman. 1983. Situational factors in disputes leading to criminal violence. Criminology 21:59-74.

Ferrándiz, Francisco, und Alejandro Baer. 2008. Digital memory: the visual recording of mass grave exhumations in contemporary Spain. Forum Qualitative Sozialforschung 9:Art. 35.

Flick, Uwe. 1992. Triangulation revisited: strategy of validation or alternative? Journal for the Theory of Social Behaviour 22:175-197.

Franzosi, Roberto. 2012. On quantitative narrative analysis. In Varieties of narrative analysis, Hrsg. James A. Holstein, Jaber F. Gubrium, 75-98. Los Angeles: SAGE.

Gatehouse, Gabriel. 2013. "Kenya Military Names Westgate Mall Attack Suspects." BBC News, 15.10.2013.

Gong, Abe. 2011. An automated snowball census of the political web. SSRN Electronic Journal, https:// doi.org/10.2139/ssrn.1832024.

Goodwin, Charles. 1994. Professional vision. American Anthropologist 96:606-633.

Gross, James J., und Robert W. Levenson. 1997. Hiding feelings: the acute effects of inhibiting negative and positive emotion. Journal of Abnormal Psychology 106:95-103.

Guera, David, und Edward J. Delp. 2018. Deepfake video detection using recurrent neural networks. In 15th IEEE International Conference on Advanced Video and Signal Based Surveillance (AVSS), 1-6. Auckland: IEEE.

Harper, Douglas. 1988. Visual sociology: expanding sociological vision. The American Sociologist 19:54-70.

Heath, Christian, Jon Hindmarsh, und Paul Luff. 2010. Video in qualitative research: analysing social interaction in everyday life. Introducing qualitative methods. Los Angeles: SAGE.

Heitmeyer, Wilhelm, und John Hagan. 2002. Gewalt. Zu den Schwierigkeiten einer systematischen Bestandsaufnahme. In Internationales Handbuch der Gewaltforschung, Hrsg. Wilhelm Heitmeyer, John Hagan, 15-25. Wiesbaden: Westdeutscher Verlag.

Hess, Ursula. 2019. Applications, opportunities, and challenges of 21st century video data in psychology. Vortrag, Blankensee-Colloquium on Capturing and Analyzing Social Change, Berlin.

Hoebel, Thomas, und Stefan Malthaner. 2019. Über dem Zenit. Grenzen und Perspektiven der situationistischen Gewaltforschung. Mittelweg 36(28):3-14.

King, Gary, Robert O. Keohane, und Sidney Verba. 1994. Designing social inquiry: scientific inference in qualitative research. Princeton: Princeton University Press.

Klusemann, Stefan. 2009. Atrocities and confrontational tension. Frontiers in Behavioral Neuroscience 3:1-10.

Klusemann, Stefan. 2012. Massacres as process: a micro-sociological theory of internal patterns of mass atrocities. European Journal of Criminology 9:468-480.

Knöbl, Wolfgang. 2019. Collins im Kontext. Zur Vorgeschichte der jüngeren Gewaltsoziologie. Mittelweg 36(28):15-39.

Knoblauch, Hubert, Alejandro Baer, Eric Laurier, Sabine Petschke, und Bernt Schnettler. 2008. Visual analysis. New developments in the interpretative analysis of video and photography. Forum Qualitative Sozialforschung 9:Art. 14.

Kuckartz, Udo. 2014. Mixed Methods: Methodologie, Forschungsdesigns und Analyseverfahren. Wiesbaden: Springer VS.

Lazer, David, Ryan Kennedy, Gary King, und Alessandro Vespignani. 2014. The parable of Google flu: traps in big data analysis. Science 343:1203-1205.

Legewie, Nicolas M. 2013. An introduction to applied data analysis with qualitative comparative analysis (QCA). Forum Qualitative Sozialforschung 14:Art 15.

Legewie, Nicolas M., und Anne Nassauer. 2018. Youtube, Google, Facebook: 21st century online video research and research ethics. Forum Qualitative Sozialforschung 19:Art. 32.

Legewie, Nicolas M. 2019. Web crawling and scraping for video data analysis. Vortrag, Blankensee-Colloquium on Capturing and Analyzing Social Change, Berlin.

Legewie, Nicolas M., und Anne Nassauer. 2020. Livestreams as video data? The 2019 Christchurch attack livestream as a case study in research ethics. In Legal and ethical issues of live streaming, Hrsg. Shing-Ling S. Chen. Oxford: Lexington Books.

Legewie, Nicolas M., Anne Nassauer, und Malika Stuerznickel. 2019. Potentiale der Analyse Visueller Daten im 21. Jahrhundert. Bericht des Blankensee-Kolloquiums 2019 zur Erfassung und Analyse Sozialen Wandels. Blankensee-Colloquium, Berlin.

Levine, Mark, Paul J. Taylor, und Rachel Best. 2011. Third parties, violence, and conflict resolution: the role of group size and collective action in the microregulation of violence. Psychological Science 22:406-412. 
Liebst, Lasse, Richard Philpot, Wim Bernasco, Kasper Dausel, Peter Ejbye-Ernst, Mathias Nicolaisen, und Marie Lindegaard. 2019. Social relations and presence of others predict bystander intervention: Evidence from violent incidents captured on CCTV. Aggressive Behavior 45:598-609.

Magdy, Walid, Kareem Darwish, und Ingmar Weber. 2015. Failedrevolutions: using twitter to study the antecedents of ISIS support. arXiv:1503.02401 [physics].

Markham, Annette, und Elizabeth A. Buchanan. 2012. Ethical decision-making and Internet research: recommendations from the AoIR ethics working committee (version 2.0). Association of Internet $\mathrm{Re}-$ searchers. https://aoir.org/reports/ethics2.pdf

Mastrofski, Stephen D., Roger B. Parks, und John D. McCluskey. 2010. Systematic social observation in criminology. In Handbook of quantitative criminology, Hrsg. Alex R. Piquero, David Weisburd, 225-247. New York: Springer.

McCluskey, John D., Craig D. Uchida, Shellie E. Solomon, Christine Connor, und Lauren Revier. 2019. Assessing the effects of body-worn cameras on procedural justice in the Los angeles police department. Criminology 57:208-236.

McFarland, Daniel A., Daniel Ramage, Jason Chuang, Jeffrey Heer, Christopher D. Manning, und Daniel Jurafsky. 2013. Differentiating language usage through topic models. Poetics, Topic Models and the Cultural Sciences 41:607-625.

Merz, Nicolas, Sven Regel, und Jirka Lewandowski. 2016. The manifesto corpus: a new resource for research on political parties and quantitative text analysis. Research \& Politics 3:1-8.

Moritz, Christine, und Michael Corsten (Hrsg.). 2018. Handbuch Qualitative Videoanalyse. Handbuch. Wiesbaden: Springer VS.

Mosselman, Floris, Don Weenink, und Marie Rosenkrantz Lindegaard. 2018. Weapons, body postures, and the quest for dominance in robberies: a qualitative analysis of video footage. Journal of Research in Crime and Delinquency 55:3-26.

Nassauer, Anne. 2016. From peaceful marches to violent clashes: a micro-situational analysis. Social Movement Studies 15:1-16.

Nassauer, Anne. 2018a. How robberies succeed or fail: analyzing crime caught on CCTV. Journal of Research in Crime and Delinquency 55:125-154.

Nassauer, Anne. 2018b. Situational dynamics and the emergence of violence during protests. Psychology of Violence 8:293-304.

Nassauer, Anne. 2019a. Documenting dissent: US mainstream media reports versus activists' capture and representation of protests. In American counter/publics, Hrsg. Ulla Haselstein, Frank Kelleter, Alexander Starre, und Birte Wege, 117-136. Heidelberg: Universitätsverlag Winter.

Nassauer, Anne. 2019b. Situational breakdowns - understanding protest violence and other surprising outcomes. Oxford: Oxford University Press.

Nassauer, Anne. 2020. Racism, interaction dynamics and police shootings in the United States. Konferenzartikel, Eastern Sociological Society Annual Meeting, Februar 2020, Panel "Racism and Resistance", Philadelphia.

Nassauer, Anne, und Nicolas M. Legewie. 2019. Analyzing 21st century video data on situational dynamics -issues and challenges in video data analysis. Social Sciences 8:100.

Nassauer, Anne, und Nicolas Legewie. 2018. Video data analysis: a methodological frame for a novel research trend. Sociological Methods \& Research, https://doi.org/10.1177/0049124118769093.

Norris, Sigrid. 2004. Analyzing multimodal interaction: a methodological framework. New York: Routledge.

Pauwels, Luc. 2006. Ethical issues of online (visual) research. Visual Anthropology 19:365-369.

Pauwels, Luc. 2010. Visual sociology reframed: an analytical synthesis and discussion of visual methods in social and cultural research. Sociological Methods \& Research 38:545-581.

Pauwels, Luc. 2015. Reframing visual social science: towards a more visual sociology and anthropology. Cambridge: Cambridge University Press.

Philpot, Richard, und Mark Levine. 2016. Street violence as a conversation: using CCTV footage to explore the dynamics of violent episodes. Konferenzartikel, American Society for Criminology Annual Meeting, New Orleans.

Philpot, Richard, Lasse Suonperä Liebst, Mark Levine, Wim Bernasco, und Marie Rosenkrantz Lindegaard. 2019. Would I be helped? Cross-national CCTV footage shows that intervention is the norm in public conflicts. American Psychologist 75:66-75.

Ragin, Charles C. 2008. Redesigning social inquiry: fuzzy sets and beyond. Chicago: University of Chicago Press.

Rat, S.W. 2017. Forschungsethische Grundsätze und Prüfverfahren in den Sozial- und Wirtschaftswissenschaften. 9. RatSWD Output Series. Berlin: Rat für Sozial- und Wirtschaftsdaten (RatSWD). 
Rosenkrantz Lindegaard, Marie, Wim Bernasco, und Scott Jacques. 2015. Consequences of expected and observed victim resistance for offender violence during robbery events. Journal of Research in Crime and Delinquency 52:32-61.

Russakovsky, Olga. 2019. Automated analysis of video data using computer vision. Vortrag, BlankenseeColloquium on Capturing and Analyzing Social Change, Berlin.

Salganik, Matthew J. 2018. Bit By Bit: Social resarch in the digital age. New Jersey: Princeton University Press.

Schwartz, H. Andrew, und Lyle H. Ungar. 2015. Data-driven content analysis of social media: a systematic overview of automated methods. The ANNALS of the American Academy of Political and Social Science 659:78-94.

Schwartz, H. Andrew, Johannes C. Eichstaedt, Margaret L. Kern, Lukasz Dziurzynski, Stephanie M. Ramones, Megha Agrawal, Achal Shah, Michal Kosinski, David Stillwell, M. Artin E.P. Seligman, und Lyle H. Ungar. 2013. Personality, gender, and age in the language of social media: the open-vocabulary approach. PLOS ONE 8:e73791.

Sigurdsson, Gunnar A., Olga Russakovsky, Ali Farhadi, Ivan Laptev, und Abhinav Gupta. 2016. Much ado about time: exhaustive annotation of temporal data. arXiv:1607.07429.

Sigurdsson, Gunnar A., Olga Russakovsky, und Abhinav Gupta. 2017. What actions are needed for understanding human actions in videos? In 2017 IEEE International Conference on Computer Vision (ICCV), 2156-2165.

Small, Mario Luis. 2011. How to conduct a mixed methods study: recent trends in a rapidly growing literature. Annual Review of Sociology 37:57-86.

Stott, Clifford, und Steven Reicher. 1998. How conflict escalates: the inter-group dynamics of collective football crowd "violence". Sociology 32:353-377.

Tuma, René. 2017. Spurensuche und Beweis - Videoanalyse in der Polizeiarbeit. In Videoprofis im Alltag, Hrsg. René Tuma, 129-175. Wiesbaden: Springer VS.

Uchida, Craig. 2019. Systematic social observations and video footage: a comparative analysis of policecitizen encounters. Vortrag, Blankensee-Colloquium on Capturing and Analyzing Social Change, Berlin.

University, Stanford. 2009. Dynamics of collective action dataset. http://www.stanford.edu/group/ collectiveaction/cgi-bin/drupal/. Zugegriffen: 17. Febr. 2020.

Vrij, Aldert, Lorraine Hope, und Ronald P. Fisher. 2014. Eliciting reliable information in investigative interviews. Policy Insights from the Behavioral and Brain Sciences 1:129-136.

Wagner, Jon. 2002. Contrasting images, complementary trajectories: sociology, visual sociology and visual research. Visual Studies 17:160-171.

Watson, Patrick G. 2018. The documentary method of [video] interpretation: a paradoxical verdict in a police-involved shooting and its consequences for understanding crime on camera. Human Studies 41:121-135.

Wiles, Rose, Andrew Clark, und Jon Prosser. 2011. Visual research ethics at the crossroads. In The SAGE handbook of visual research methods, Hrsg. Eric Margolis, Luc Pauwels, 685-706. Los Angeles: SAGE.

Wiles, Rose, Jon Prosser, Anna Bagnoli, Andrew Clark, Katherine Davies, Sally Holland, und Emma Renold. 2008. Visual ethics: ethical issues in visual research. Working paper. http://eprints.ncrm.ac. uk/421/. Zugegriffen: 17. Febr. 2020.

Wissenschaftszentrum Berlin für Sozialforschung. 2011. PRODAT - Dokumentation und Analyse von Protestereignissen in der Bundesrepublik. http://www.wzb.eu/de/forschung/beendete-forschungs programme/zivilgesellschaft-und-politische-mobilisierung/projekte/prodat-dokument. Zugegriffen: 17. Febr. 2020.

Wright, Richard, und Scott H. Decker. 1997. Armed robbers in action: stickups and street culture. Lebanon: University Press New England.

Yeung, Serena, Olga Russakovsky, Jin Ning, Mykhaylo Andriluka, Greg Mori, und Li Fei-Fei. 2015. Every moment counts: dense detailed labeling of actions in complex videos. arXiv:1507.05738 [cs.CV].

Zimmer, Michael. 2010. But the data is already public: on the ethics of research in Facebook. Ethics and Information Technology 12:313-325.

Anne Nassauer ist Juniorprofessorin für Soziologie am John-F-Kennedy Institut der Freien Universität Berlin und aktuell Gastwissenschaftlerin des Instituts für Soziologie an der Yale University (2019 \& 2020). Zu Ihren Forschungsschwerpunkten gehören kollektives Handeln, Gewalt und Interaktionsroutinen, Polizei und Kriminalität, sowie Forschungsmethoden (u. a. im Bereich der der Videoanalyse). 
Nicolas M. Legewie ist Gastwissenschaftler am Institut für Soziologie der University of Pennsylvania. Er forscht und lehrt zu den Themen soziale Mobilität, soziale Netzwerke und Bildung, sowie Forschungsmethoden (Videodatenanalyse, Mixed Methods, digitale Sozialforschung, qualitative Interviewführung und qualitative comparative analysis, QCA). 